Revista de MATEMÁtica: TeORÍA y APliCACIONEs 2018 25(1) : 151-168

CIMPA - UCR ISSN: 1409-2433 (PRINT), 2215-3373 (ONLINE)

\title{
EXISTENCIA DE LA SOLUCIÓN DÉBIL DE UN MODELO DE DIFUSIÓN ESTRATIFICADA VÍA UN MÉTODO ITERATIVO
}

\section{EXISTENCE OF THE WEAK SOLUTION FOR A STRATIFIED DIFFUSION MODEL VIA AN ITERATIVE METHOD}

\author{
Ricardo CANo Macías* Jorge Mauricio Ruiz V. ${ }^{\dagger}$
}

Received: 7/Jun/2017; Revised: 5/Dec/2017;

Accepted: 6/Dec/2017

Revista de Matemática: Teoría y Aplicaciones is licensed under a Creative Commons Reconocimiento-NoComercial-Compartirigual 4.0 International License. Creado a partir de la obra en http://www.revistas.ucr.ac.cr/index.php/matematica

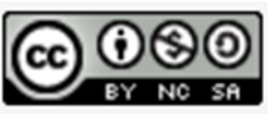

*Facultad de Ingeniería, Universidad de La Sabana, Chía, Colombia. E-Mail: ricardocm@unisabana.edu.co

${ }^{\dagger}$ Departamento de Matemáticas, Universidad Nacional de Colombia, Bogotá D.C., Colombia. E-Mail: jmruizv@unal.edu.co 


\title{
Resumen
}

Se estudia la existencia y unicidad de la solución débil de un problema de difusión estratificada no lineal. Para ésto, se construye un método alternativo basado en sustituciones sucesivas de una aproximación lineal del problema original. Empleando la teoría de ecuaciones diferenciales parciales y usando inducción matemática se prueba que cada uno de los problemas lineales de la iteración tiene una única solución débil, obteniendo así, una sucesión de soluciones débiles. Finalmente, se demuestra que dicha suseción es de Cauchy y que converge a la solución débil del problema.

Palabras clave: solución débil; método iterativo; difusión no lineal.

\begin{abstract}
We study the existence and uniqueness of the solution of a non-linear stratified diffusion problem. To this aim, we construct an alternative method based on successive substitutions of a linear approximation of the original problem. We use the theory of partial differential equations and mathematical induction to prove that each of the linear problems of the iteration has a unique weak solution. Finally, we prove that the sequence of weak solutions obtained is a Cauchy sequence that converges to the weak solution of the problem.
\end{abstract}

Keywords: weak solution; Iterative method; non-linear diffusion.

Mathematics Subject Classification: 35D30, 35A07, 35K55, 35Q92.

\section{Introducción}

El objetivo central de este trabajo consiste en el estudio de la existencia y unicidad de la solución débil del problema de difusión no lineal

$$
\left\{\begin{array}{l}
u_{t}-\nabla \cdot\left(\left(D_{0}+\left(D_{1}-D_{0}\right) \frac{u^{\gamma}}{u^{\gamma}+A}\right) \nabla u\right)=u(1-u) \text { en } \Omega_{T} \\
u=0 \text { en } \partial \Omega \\
u=u_{0} \text { en } \Omega \times\{t=0\}
\end{array}\right.
$$

donde $\Omega \subset \mathbb{R}^{n}(n=1,2)$ es un subconjunto abierto, acotado y suficientemente regular y $\Omega_{T}:=\Omega \times(0, T]$ para algún tiempo fijo $T>0$. 
El coeficiente de difusión no lineal $\mathcal{H}(u):=D_{0}+\left(D_{1}-D_{0}\right) \frac{u^{\gamma}}{u^{\gamma}+A}$, con $\gamma>1, D_{0}$ y $D_{1}$ constantes positivas, describe el movimiento espontaneo e independiente de la población $u$ el cual se incrementa a causa de la presión demográfica [1]. Más precisamente, la difusión sucede de manera estratificada, es decir, en tres etapas: una etapa de asentamiento, una de diseminación y una de saturación [9].

El problema (1) modela la dispersión en tiempo y espacio de especies biológicas, en la que los individuos colonizadores establecen nuevas colonias cercanas a su asentamiento original y que a corto plazo se fusionan. Especies que se propagan de esta forma son por ejemplo: insectos como el gorgojo del arroz, aves como el camachuelo mexicano en Norte América y el estornino pinto europeo [11].

Con $\mathcal{H}(u)=1$, el problema (1) se reduce a la ecuación de Fischer [6] ampliamente estudiada. Por otra parte, si se considera el problema homogéneo y $\mathcal{H}(u)=u^{\gamma}$, el problema (1) coincide con la ecuación de medios porosos usada en diversas áreas como flujo de gases en medios porosos, dinámica de fluidos incompresibles, transferencia de calor no lineal y procesamiento de imágenes [15] y que también ha sido empleada en el estudio de la difusión de poblaciones biológicas [7] [8] y [16].

La existencia y unicidad de la solución del problema (1) puede abordarse empleando varias técnicas, como las técnicas de semigrupos [13] o mediante técnicas de monotonía [14], [17]. Sin embargo las técnicas de monotonía conducen a formular hipótesis sobre los coeficientes de la ecuación que tienen muy poco sentido desde el punto de vista biológico. Por esta razón, para el análisis del problema (1), se propone un método alternativo basado en sustituciones sucesivas de una aproximación lineal del problema. Luego, se emplea la teoría de ecuaciones diferenciales parciales lineales y usando inducción matemática se prueba que cada uno de los problemas lineales de la iteración tiene una única solución débil, obteniendo así, una sucesión de soluciones débiles la cual es acotada uniformemente. Finalmente, de las propiedades de los espacios de Bochner se demuestra que dicha suseción es de Cauchy y que converge a la solución débil del problema (1).

Para efectos de completitud y claridad, en el apéndice del artículo se resumen las desigualdes y teoremas que se emplean en nuestro análisis. Además, la notación que empleamos es la que aparece en [5]. Antes de enunciar nuestro principal resutado, daremos la definición de una solución débil. 
Definición 1 Una función

$$
u \in L^{\infty}\left(0, T ; H_{0}^{1}(\Omega)\right), \text { con } u_{t} \in L^{2}\left(0, T ; H_{0}^{1}(\Omega)\right),
$$

es una solución débil del problema (1) siempre que

(i) $\int_{\Omega} u_{t} \phi d x+\int_{\Omega} \mathcal{H}(u) \nabla u \nabla \phi d x=\int_{\Omega} u(1-u) \phi d x$ para todo $\phi \in H_{0}^{1}(\Omega)$ $y$ casi todo $t \in[0, T], y$

(ii) $u(x, 0)=u_{0}$.

El principal resultado en este artículo es el siguiente teorema.

Teorema 1 Sea $\Omega \subset \mathbb{R}^{n}(n=1,2)$ un subconjunto abierto, acotado y suficientemente regular. $\Omega_{T}:=\Omega \times(0, T]$ para algún tiempo fijo $T>0$ y $u_{0}(x) \in H^{2}(\Omega), 0 \leq u_{0}(x) \leq 1$. Existe una única solución débil $u(x, t)$ del problema $(1)$.

\section{Método iterativo}

Se abordará la demostración del teorema de la siguiente manera: Primero, se construye una sucesión iterativa de modificaciones lineales del problema (1) de la forma

$$
\begin{cases}u_{t}^{0}-\nabla \cdot\left(\mathcal{H}\left(u_{0}\right) \nabla u^{0}\right)=0 & \text { en } \Omega_{T} \\ u^{0}=0 & \text { en } \partial \Omega \\ u^{0}=u_{0} & \text { en } \Omega \times\{t=0\}\end{cases}
$$

y para $k \geq 0$

$$
\begin{cases}u_{t}^{k+1}-\nabla \cdot\left(\mathcal{H}\left(u^{k}\right) \nabla u^{k+1}\right)=u^{k}\left(1-u^{k}\right) & \text { en } \Omega_{T} \\ u^{k+1}=0 & \text { en } \partial \Omega \\ u^{k+1}=u_{0} & \text { en } \Omega \times\{t=0\} .\end{cases}
$$

Segundo, explotando los resultados bien conocidos de la teoría de ecuaciones diferenciales parciales lineales [5], se prueba la existencia y unicidad de la solución débil del problema (2) y mediante inducción matemática, la existencia y unicidad de la solución débil del problema (3) para cualquier iteración $k$. Por último, se constata el criterio de Cauchy para sucesiones y así demostrar la convergencia de la sucesión iterativa de soluciones débiles. 
Lema 1 Bajo las condiciones del teorema 1, existe una única solución débil $u^{0}$ del problema (2), tal que

$$
\begin{aligned}
& u^{0} \in L^{\infty}\left(0, T ; H^{2}(\Omega)\right), \\
& u_{t}^{0} \in L^{\infty}\left(0, T ; L^{2}(\Omega)\right) \cap L^{2}\left(0, T ; H_{0}^{1}(\Omega)\right)
\end{aligned}
$$

y

$$
\underset{0 \leq t \leq T}{\operatorname{ess} \sup }\left(\left\|u^{0}(t)\right\|_{H^{2}(\Omega)}+\left\|u_{t}^{0}\right\|_{L^{2}(\Omega)}\right)+\left\|u_{t}^{0}\right\|_{L^{2}\left(0, T ; H_{0}^{1}(\Omega)\right)} \leq C\left\|u_{0}\right\|_{H^{2}(\Omega)}
$$

para alguna constante $C$ apropiada que depende de $\Omega, T$ y $\mathcal{H}\left(u_{0}\right)$.

Demostración. Existencia. Claramente,

$$
D_{0} \leq \mathcal{H}\left(u_{0}\right) \leq D_{0}+\frac{D_{1}-D_{0}}{A}
$$

Del Teorema 5, §7.1.3, en [5], existe una solución débil $u^{0}$ del problema (2).

Unicidad. Supongamos que $u^{0}$ y $v^{0}$ son dos soluciones débiles del problema (2). Es suficiente ver que $w^{0}=u^{0}-v^{0}=0$ en casi toda parte en $\Omega_{T}$. Al reemplazar $w^{0}$ en la formulación débil del problema (2), resulta

$$
\begin{gathered}
\int_{\Omega} w_{t}^{0} w^{0} d x+\int_{\Omega} \mathcal{H}\left(u_{0}\right) \nabla w^{0} \nabla w^{0} d x=0 \\
\frac{1}{2} \frac{d}{d t}\left\|w^{0}\right\|_{L^{2}(\Omega)}^{2}=-\int_{\Omega} \mathcal{H}\left(u_{0}\right)\left(\nabla w^{0}\right)^{2} d x,
\end{gathered}
$$

pero por el Teorema 2, §6.2.2, en [5], existen constantes $\alpha \geq 0$ y $\beta \geq 0$, tal que

$$
\int_{\Omega} \mathcal{H}\left(u_{0}\right)\left(\nabla w^{0}\right)^{2} d x \geq \beta\left\|w^{0}\right\|_{H^{1}(\Omega)}^{2}-\alpha\left\|w^{0}\right\|_{L^{2}(\Omega)}^{2} \geq-\alpha\left\|w^{0}\right\|_{L^{2}(\Omega)}^{2} .
$$

Por consiguiente

$$
\frac{d}{d t}\left\|w^{0}\right\|_{L^{2}(\Omega)}^{2} \leq 2 \alpha\left\|w^{0}\right\|_{L^{2}(\Omega)}^{2} .
$$

De la desigualdad de Gronwall y (7) se sigue

$$
\left\|w^{0}\right\|_{L^{2}(\Omega)}^{2} \leq\left\|w^{0}(0)\right\|_{L^{2}(\Omega)}^{2} e^{\int_{0}^{T} 2 \alpha d t} .
$$

Como $w^{0}(0)=0$, entonces $w^{0}(x, t)=0$ en casi toda parte de $\Omega_{T}$. Además, del Teorema 5, §7.1.3, en [5], se satisfacen (4) y (5). 
Lema 2 La solución débil $u^{0}$ del problema (2) satisface $0 \leq u^{0}(x, t) \leq 1$ en casi toda parte $\Omega_{T}$.

Demostración. Probemos que $u^{0}(x, t) \geq 0$. Sea $\phi=\left(u^{0}\right)^{-}:=\min \left\{0, u^{0}\right\}$ una función de prueba. Al sustituir $\phi$ en la formulación débil del problema (2), se obtiene

$$
\begin{aligned}
& \int_{\Omega} u_{t}^{0}\left(u^{0}\right)^{-} d x+\int_{\Omega} \mathcal{H}\left(u_{0}\right) \nabla u^{0} \nabla\left(u^{0}\right)^{-} d x=0 \\
& \frac{1}{2} \frac{d}{d t} \int_{\Omega}\left|\left(u^{0}\right)^{-}\right|^{2} d x+\int_{\Omega} \mathcal{H}\left(u_{0}\right)\left|\nabla\left(u^{0}\right)^{-}\right|^{2} d x=0 .
\end{aligned}
$$

Puesto que, $0 \leq D_{0} \leq \mathcal{H}\left(u_{0}\right) \leq D_{0}+\frac{D_{1}-D_{0}}{A}$ entonces

$$
\frac{1}{2} \frac{d}{d t} \int_{\Omega}\left|\left(u^{0}\right)^{-}\right|^{2} d x=-\int_{\Omega} \mathcal{H}\left(u_{0}\right)\left|\nabla\left(u^{0}\right)^{-}\right|^{2} d x \leq 0 .
$$

Luego, para alguna constante $\beta_{0} \geq 0$, se tiene

$$
\frac{1}{2} \frac{d}{d t}\left\|\left(u^{0}\right)^{-}\right\|_{L^{2}(\Omega)}^{2} \leq \beta_{0}\left\|\left(u^{0}\right)^{-}\right\|_{L^{2}(\Omega)}^{2} .
$$

De la desigualdad de Gronwall y (8), se tiene

$$
\left\|\left(u^{0}\right)^{-}\right\|_{L^{2}(\Omega)}^{2} \leq\left\|\left(u^{0}\right)^{-}(0)\right\|_{L^{2}(\Omega)}^{2} e^{\int_{0}^{T} \beta_{0} d t} .
$$

Dado que $\left(u^{0}\right)^{-}(0):=\min \left\{0, u_{0}\right\}$ y $u_{0}>0$, entonces $\left(u^{0}\right)^{-}(0)=0$. De (9), se deduce que $\left(u^{0}\right)^{-}=0$. Por lo tanto, $u^{0}(x, t) \geq 0$ en casi toda parte de $\Omega_{T}$.

Ahora se prueba que $u^{0}(x, t) \leq 1$. Sea $\phi=\left(u^{0}-1\right)^{+}:=\max \left\{\left(u^{0}-1\right), 0\right\}$. Al sustituir $\phi$ en la formulación débil del problema (2), se obtiene

$$
\begin{aligned}
& \int_{\Omega} u_{t}^{0}\left(u^{0}-1\right)^{+} d x+\int_{\Omega} \mathcal{H}\left(u_{0}\right) \nabla u^{0} \nabla\left(u^{0}-1\right)^{+} d x=0 \\
& \frac{1}{2} \frac{d}{d t} \int_{\Omega}\left|\left(u^{0}-1\right)^{+}\right|^{2} d x+\int_{\Omega} \mathcal{H}\left(u_{0}\right)\left|\nabla\left(u^{0}-1\right)^{+}\right|^{2} d x=0 \\
& \frac{1}{2} \frac{d}{d t}\left\|\left(u^{0}-1\right)^{+}\right\|_{L^{2}(\Omega)}^{2}=-\int_{\Omega} \mathcal{H}\left(u_{0}\right)\left|\nabla\left(u^{0}-1\right)^{+}\right|^{2} d x \leq 0 .
\end{aligned}
$$

Así,

$$
\frac{1}{2} \frac{d}{d t}\left\|\left(u^{0}-1\right)^{+}\right\|_{L^{2}(\Omega)}^{2} \leq \beta_{1}\left\|\left(u^{0}-1\right)^{+}\right\|_{L^{2}(\Omega)}^{2}
$$


para alguna constante $\beta_{1} \geq 0$. De la desigualdad de Gronwall y (10), resulta

$$
\left\|\left(u^{0}-1\right)^{+}\right\|_{L^{2}(\Omega)}^{2} \leq\left\|\left(u^{0}-1\right)^{+}(0)\right\|_{L^{2}(\Omega)}^{2} e^{\int_{0}^{T} \beta_{1} d t} .
$$

Como $\left(u^{0}-1\right)^{+}(0):=\max \left\{0,\left(u_{0}-1\right)\right\}$, entonces $\left(u^{0}-1\right)^{+}(0)=0$, pues $0 \leq u_{0} \leq 1$. Lo que implica que $\left(u^{0}-1\right)^{+}(x, t)=0$. Por lo tanto, $u^{0}(x, t) \leq 1$ en casi toda parte de $\Omega_{T}$.

\subsection{Prueba por inducción}

Los lemas 1 y 2 , proporcionan el valor inicial $u^{0}(x, t)$ apropiado para generar a partir del problema (3), con $k=0,1,2, \ldots$, una sucesión de soluciones convergente a la solución del problema (1). A continuación mediante inducción matemática, se prueba que la sucesión generada satisface el siguiente lema.

Lema 3 Para todo $k \in \mathbb{N}_{0}$, el probema (3) tiene una única solución débil $u^{k}(x, t), 0 \leq u^{k}(x, t) \leq 1$ tal que:

$$
\begin{aligned}
& u^{k} \in L^{\infty}\left(0, T ; H^{2}(\Omega)\right), \\
& u_{t}^{k} \in L^{\infty}\left(0, T ; L^{2}(\Omega)\right) \cap L^{2}\left(0, T ; H_{0}^{1}(\Omega)\right)
\end{aligned}
$$

$y$

$$
\begin{aligned}
\underset{0 \leq t \leq T}{\operatorname{ess} \sup }\left(\left\|u^{k}(t)\right\|_{H^{2}(\Omega)}+\|\right. & \left.u_{t}^{k} \|_{L^{2}(\Omega)}\right)+\left\|u_{t}^{k}\right\|_{L^{2}\left(0, T ; H_{0}^{1}(\Omega)\right)} \\
& \leq C\left\|u_{0}\right\|_{H^{2}(\Omega)}+\left\|f\left(u^{k-1}\right)\right\|_{H^{1}\left(0, T ; L^{2}(\Omega)\right)}
\end{aligned}
$$

para alguna constante $C$ apropiada que depende de $\Omega, T$ y $\mathcal{H}\left(u^{k-1}\right)$.

\section{Demostración.}

Inicio de la inducción: Teniendo en cuenta las propiedades del primer término $u^{0}(x, t)$ de la sucesión (lema 1 y 2 ), la prueba para el caso $k=0$ sigue las mismas ideas de la demostración de los lemas 4 y 5.

Hipótesis de inducción: Supongamos que el lema es válido para $n=k$.

Paso inductivo: Probemos que para $n=k+1$, se satisfacen los lemas 4 y 5 .

Lema 4 Bajo la hipótesis de inducción, existe una única solución débil $u^{k+1}$ del problema (3). 
Demostración. Es suficiente ver que $\mathcal{H}\left(u^{k}\right) \in L^{\infty}\left(\Omega_{T}\right)$ y $f_{t}\left(u^{k}\right) \in$ $L^{2}\left(0, T ; L^{2}(\Omega)\right)$, donde $f\left(u^{k}\right):=u^{k}\left(1-u^{k}\right)$. Como en (6), se tiene que $\mathcal{H}\left(u^{k}\right)$ es acotada.

Veamos ahora que $f_{t}\left(u^{k}\right) \in L^{2}\left(0, T ; L^{2}(\Omega)\right)$. Por hipótesis de inducción, resulta

$$
\begin{aligned}
\int_{0}^{T} \int_{\Omega}\left|f_{t}\left(u^{k}\right)\right|^{2} d x d t & =\int_{0}^{T} \int_{\Omega}\left|\frac{\partial f}{\partial u^{k}} u_{t}^{k}\right|^{2} d x d t \\
& =\int_{0}^{T} \int_{\Omega}\left(1-2 u^{k}\right)^{2}\left(u_{t}^{k}\right)^{2} d x d t \\
& \leq \int_{0}^{T} \int_{\Omega}\left|u_{t}^{k}\right|^{2} d x d t \\
& \leq \int_{0}^{T}\left\|u_{t}^{k}\right\|_{L^{2}(\Omega)}^{2} d t \\
& \leq C\left\|u_{0}\right\|_{H^{2}(\Omega)}^{2} .
\end{aligned}
$$

Así, del Teorema 5, §7.1.3, en [5], existe una única solución débil $u^{k+1}$ del problema (3), para cada $k \in \mathbb{N}_{0}$ y se satisfacen (11) y (12).

Lema 5 La solución débil $u^{k+1}$ del problema (3) satisface $0 \leq u^{k+1}(x, t) \leq 1$ en casi toda parte $\Omega_{T}$.

Demostración. Probemos que $u^{k+1}(x, t) \geq 0$. Definamos $\phi=\left(u^{k+1}\right)^{-}:=$ $\min \left\{0, u^{k+1}\right\}$. Al reemplazar $\phi$ en la formulación débil de (3), resulta

$$
\int_{\Omega} u_{t}^{k+1}\left(u^{k+1}\right)^{-} d x+\int_{\Omega} \mathcal{H}\left(u^{k}\right) \nabla u^{k+1} \nabla\left(u^{k+1}\right)^{-} d x=\int_{\Omega} u^{k}\left(1-u^{k}\right)\left(u^{k+1}\right)^{-} d x .
$$

Como $\int_{\Omega} u^{k}\left(1-u^{k}\right)\left(u^{k+1}\right)^{-} d x \leq 0$, entonces

$$
\begin{aligned}
\frac{1}{2} \frac{d}{d t}\left\|\left(u^{k+1}\right)^{-}\right\|_{L^{2}(\Omega)}^{2} & \leq-\int_{\Omega} \mathcal{H}\left(u^{k}\right)\left[\nabla\left(u^{k+1}\right)^{-}\right]^{2} d x \\
& \leq \beta_{2}\left\|\left(u^{k+1}\right)^{-}\right\|_{L^{2}(\Omega)}^{2}
\end{aligned}
$$

para alguna constante $\beta_{2} \geq 0$. De la desigualdad de Gronwall y (13), se sigue

$$
\left\|\left(u^{k+1}\right)^{-}\right\|_{L^{2}(\Omega)}^{2} \leq\left\|\left(u^{k+1}\right)^{-}(0)\right\|_{L^{2}(\Omega)}^{2} e^{\int_{0}^{T} 2 \beta_{2} d t} .
$$


Ya que $\left(u^{k+1}\right)^{-}(0):=\min \left\{0, u_{0}\right\}$ y $0 \leq u_{0} \leq 1$, entonces $\left(u^{k+1}\right)^{-}(0)=0$. Así, de (14) se sigue que $u^{k+1}(x, t) \geq 0$ casi en toda parte de $\Omega_{T}$.

Probemos que $u^{k+1}(x, t) \leq 1$. Sea $\phi=\left(u^{k+1}-1\right)^{+}:=\max \left\{0,\left(u^{k+1}-1\right)\right\}$. $\mathrm{Al}$ sustituir $\phi$ en la formulación débil de (3), se obtiene

$$
\begin{aligned}
\int_{\Omega} u_{t}^{k+1}\left(u^{k+1}-1\right)^{+} d x+\int_{\Omega} \mathcal{H}\left(u^{k}\right) & \nabla u^{k+1} \nabla\left(u^{k+1}-1\right)^{+} d x \\
& =\int_{\Omega} u^{k}\left(1-u^{k}\right)\left(u^{k+1}-1\right)^{+} d x .
\end{aligned}
$$

Por consiguiente,

$$
\begin{aligned}
\int_{\Omega} u_{t}^{k+1}\left(u^{k+1}-1\right)^{+} d x & \leq \int_{\Omega} u^{k}\left(1-u^{k}\right)\left(u^{k+1}-1\right)^{+} d x \\
& \leq\left\|u^{k}\left(1-u^{k}\right)\right\|_{L^{2}(\Omega)}^{2}\left\|\left(u^{k+1}-1\right)^{+}\right\|_{L^{2}(\Omega)}^{2} .
\end{aligned}
$$

Así,

$$
\frac{1}{2} \frac{d}{d t}\left\|\left(u^{k+1}-1\right)^{+}\right\|_{L^{2}(\Omega)}^{2} \leq|\Omega|^{2}\left\|\left(u^{k+1}-1\right)^{+}\right\|_{L^{2}(\Omega)}^{2} .
$$

Usando la desigualdad de Gronwall y (16), se tiene

$$
\left\|\left(u^{k+1}-1\right)^{+}\right\|_{L^{2}(\Omega)}^{2} \leq\left\|\left(u^{k+1}-1\right)^{+}(0)\right\|_{L^{2}(\Omega)}^{2} e^{\int_{0}^{T} 2 \beta_{3} d t},
$$

con $\beta_{3}=|\Omega|^{2}$. Dado que, $\left(u^{k+1}-1\right)^{+}(0):=\max \left\{0,\left(u_{0}-1\right)\right\}$ y $0 \leq u_{0} \leq 1$, se deduce que $\left(u^{k+1}-1\right)^{+}(x, t)=0$. Luego $u^{k+1}(x, t) \leq 1$ en casi toda parte de $\Omega_{T}$.

\section{Existencia y unicidad}

El objetivo de esta sección es demostrar que la sucesión $\left\{u^{k}\right\}_{k \in \mathbb{N}_{0}}$ de soluciones aproximadas es de Cauchy y su límite $u$ es la solución débil del problema (1). Para ello, se consideran las iteraciones $k$ y $k+1$ del método

$$
\begin{aligned}
u_{t}^{k+1}-\nabla \cdot\left(\mathcal{H}\left(u^{k}\right) \nabla u^{k+1}\right) & =u^{k}\left(1-u^{k}\right) \\
u_{t}^{k}-\nabla \cdot\left(\mathcal{H}\left(u^{k-1}\right) \nabla u^{k}\right) & =u^{k-1}\left(1-u^{k-1}\right) .
\end{aligned}
$$

Restando las ecuaciones (17) y (18), se obtiene

$$
\begin{aligned}
\left(u^{k+1}-u^{k}\right)_{t}-\nabla \cdot\left(\mathcal{H}\left(u^{k}\right) \nabla u^{k+1}-\right. & \left.\mathcal{H}\left(u^{k-1}\right) \nabla u^{k}\right) \\
& =u^{k}\left(1-u^{k}\right)-u^{k-1}\left(1-u^{k-1}\right) .
\end{aligned}
$$


Sumando y restando el término $\nabla \cdot\left(\mathcal{H}\left(u^{k}\right) \nabla u^{k}\right)$ en (19) y tras algunos cálculos, resulta

$$
\begin{aligned}
& \left(u^{k+1}-u^{k}\right)_{t}-\nabla \cdot\left(\mathcal{H}\left(u^{k}\right) \nabla\left(u^{k+1}-u^{k}\right)\right) \\
& \quad=u^{k}\left(1-u^{k}\right)-u^{k-1}\left(1-u^{k-1}\right)+\nabla \cdot\left(\left(\mathcal{H}\left(u^{k}\right)-\mathcal{H}\left(u^{k-1}\right)\right) \nabla u^{k}\right) .
\end{aligned}
$$

Si se denota por

$$
w^{k+1}:=u^{k+1}-u^{k} \mathrm{y} f^{k}:=u^{k}\left(1-u^{k}\right),
$$

para $k=0,1, \ldots$ la ecuación (20), puede reescribirse en la forma

$$
\begin{aligned}
& \left.w_{t}^{k+1}-\nabla \cdot\left(\mathcal{H}\left(u^{k}\right) \nabla w^{k+1}\right)\right)=f^{k}-f^{k-1} \\
& w^{k+1}=0 \text { en } \partial \Omega \\
& +\nabla \cdot\left(\left(\mathcal{H}\left(u^{k}\right)-\mathcal{H}\left(u^{k-1}\right)\right) \nabla u^{k}\right) \text { en } \Omega_{T} \\
& w^{k+1}=0 \text { en } \Omega \times\{t=0\}
\end{aligned}
$$

Nótese que la formulación débil del problema (21) tiene sentido, si

$$
\left|\int_{\Omega}\left(\mathcal{H}\left(u^{k}\right)-\mathcal{H}\left(u^{k-1}\right)\right) \nabla u^{k} \nabla \phi d x\right|<\infty
$$

para todo $\phi \in H_{0}^{1}(\Omega)$. Para ello, es suficiente que $\left(\mathcal{H}\left(u^{k}\right)-\mathcal{H}\left(u^{k-1}\right)\right) \nabla u^{k} \in$ $L^{2}\left(\Omega_{T}\right)$. En efecto

$$
\begin{aligned}
& \left\|\left(\mathcal{H}\left(u^{k}\right)-\mathcal{H}\left(u^{k-1}\right)\right) \nabla u^{k}\right\|_{L^{2}\left(\Omega_{T}\right)} \\
& =\int_{0}^{T}\left\|\left(\mathcal{H}\left(u^{k}\right)-\mathcal{H}\left(u^{k-1}\right)\right)^{2} \nabla u^{k}\right\|_{L^{2}(\Omega)} d t \\
& =\int_{0}^{T} \int_{\Omega}\left|\mathcal{H}\left(u^{k}\right)-\mathcal{H}\left(u^{k-1}\right)\right|^{2}\left|\nabla u^{k}\right|^{2} d x d t \\
& \leq \int_{0}^{T} \int_{\Omega}\left|\frac{\left(D_{1}-D_{0}\right) A}{\left(A+\left(u^{k}\right)^{\gamma}\right)\left(A+\left(u^{k-1}\right)^{\gamma}\right)}\left(\left(u^{k}\right)^{\gamma}-\left(u^{k-1}\right)^{\gamma}\right)\right|^{2}\left|\nabla u^{k}\right|^{2} d x d t \\
& \leq 2\left(\frac{D_{1}-D_{0}}{A}\right)^{2} \int_{0}^{T} \int_{\Omega}\left(\left|\left(u^{k}\right)^{\gamma}\right|+\left|\left(u^{k-1}\right)^{\gamma}\right|\right)^{2}\left|\nabla u^{k}\right|^{2} d x d t \\
& \leq 4\left(\frac{D_{1}-D_{0}}{A}\right)^{2} \int_{0}^{T} \int_{\Omega}\left|\nabla u^{k}\right|^{2} d x d t \\
& \leq 4\left(\frac{D_{1}-D_{0}}{A}\right)^{2} T\left\|\nabla u^{k}\right\|_{L^{2}(\Omega)}^{2} \\
& \leq \infty .
\end{aligned}
$$


Lema 6 La sucesión $\left\{u^{k}\right\}_{k \in \mathbb{N}_{0}}$ de soluciones aproximadas es de Cauchy en $L^{\infty}\left(0, T ; H_{0}^{1}(\Omega)\right)$ y su límite u es la solución débil del problema $(1)$.

Demostración. Al reemplazar $\phi:=w^{k+1}$ en la formulación débil de (21), se tiene

$$
\begin{aligned}
& \int_{\Omega} w_{t}^{k+1} w^{k+1} d x+\int_{\Omega} \mathcal{H}\left(u^{k}\right) \nabla w^{k+1} \nabla w^{k+1} d x \\
& =\int_{\Omega}\left(f^{k}-f^{k-1}\right) w^{k+1} d x+\int_{\Omega} \nabla \cdot\left[\left(\mathcal{H}\left(u^{k}\right)-\mathcal{H}\left(u^{k-1}\right)\right) \nabla u^{k}\right] w^{k+1} d x .
\end{aligned}
$$

Al aplicar la primera fórmula de Green al segundo término de (22), resulta

$$
\begin{aligned}
& \int_{\Omega} w_{t}^{k+1} w^{k+1} d x+\int_{\Omega} \mathcal{H}\left(u^{k}\right) \nabla w^{k+1} \nabla w^{k+1} d x \\
= & \int_{\Omega}\left(f^{k}-f^{k-1}\right) w^{k+1} d x-\int_{\Omega}\left[\left(\mathcal{H}\left(u^{k}\right)-\mathcal{H}\left(u^{k-1}\right)\right] \nabla u^{k} \nabla w^{k+1} d x\right. \\
\leq & \mid \int_{\Omega}\left(f^{k}-f^{k-1}\right) w^{k+1} d x-\int_{\Omega}\left[\left(\mathcal{H}\left(u^{k}\right)-\mathcal{H}\left(u^{k-1}\right)\right] \nabla u^{k} \nabla w^{k+1} d x \mid\right. \\
\leq & \left|\int_{\Omega}\left(f^{k}-f^{k-1}\right) w^{k+1} d x\right|+\mid \int_{\Omega}\left[\left(\mathcal{H}\left(u^{k}\right)-\mathcal{H}\left(u^{k-1}\right)\right] \nabla u^{k} \nabla w^{k+1} d x \mid .\right.
\end{aligned}
$$

Usando la desigualdad de Hölder en el primer término de (23), se sigue

$$
\begin{aligned}
\int_{\Omega} w_{t}^{k+1} w^{k+1} d x+ & \int_{\Omega} \mathcal{H}\left(u^{k}\right) \nabla w^{k+1} \nabla w^{k+1} d x \\
\leq\left\|f^{k}-f^{k-1}\right\|_{L^{2}(\Omega)}\left\|w^{k+1}\right\|_{L^{2}(\Omega)} & \\
& \quad+\int_{\Omega}\left|\mathcal{H}\left(u^{k}\right)-\mathcal{H}\left(u^{k-1}\right)\right|\left|\nabla u^{k} \nabla w^{k+1}\right| d x .
\end{aligned}
$$


Además, como

$$
\begin{aligned}
\left\|f^{k}-f^{k-1}\right\|_{L^{2}(\Omega)}^{2} & =\int_{\Omega}\left|u^{k}\left(1-u^{k}\right)-u^{k-1}\left(1-u^{k-1}\right)\right|_{L^{2}(\Omega)}^{2} d x \\
& =\int_{\Omega}\left|\left(u^{k}-u^{k-1}\right)^{2}\left(1-\left(u^{k}+u^{k-1}\right)\right)^{2}\right| d x \\
& \leq \int_{\Omega}\left|u^{k}-u^{k-1}\right|^{2} d x \\
& =\left\|w^{k}\right\|_{L^{2}(\Omega)}^{2} \\
& \leq\left\|w^{k}\right\|_{H_{0}^{1}(\Omega)}^{2}
\end{aligned}
$$

y $\mathcal{H}(u)$ es Lipschitz con constante $M$, entonces al sustituir (25) en (24), se obtiene

$$
\begin{aligned}
& \int_{\Omega} w_{t}^{k+1} w^{k+1} d x+\int_{\Omega} \mathcal{H}\left(u^{k}\right) \nabla w^{k+1} \nabla w^{k+1} d x \\
& \quad \leq\left\|w^{k}\right\|_{L^{2}(\Omega)}\left\|w^{k+1}\right\|_{L^{2}(\Omega)}+\int_{\Omega} M\left|u^{k}-u^{k-1}\right|\left|\nabla u^{k}\right|\left|\nabla w^{k+1}\right| d x .
\end{aligned}
$$

De nuevo, por la desigualdad de Hölder al segundo término de (27), se sigue

$$
\begin{aligned}
& \int_{\Omega} w_{t}^{k+1} w^{k+1} d x+\int_{\Omega} \mathcal{H}\left(u^{k}\right) \nabla w^{k+1} \nabla w^{k+1} d x \\
& \quad \leq\left\|w^{k}\right\|_{L^{2}(\Omega)}\left\|w^{k+1}\right\|_{L^{2}(\Omega)}+M\left\|w^{k} \nabla u^{k}\right\|_{L^{2}(\Omega)}\left\|\nabla w^{k+1}\right\|_{L^{2}(\Omega)} .
\end{aligned}
$$

Dado que

$$
\begin{aligned}
\frac{1}{2} \frac{d}{d t}\left\|w^{k+1}\right\|_{L^{2}(\Omega)}^{2}+D_{0}\left\|\nabla w^{k+1}\right\|_{L^{2}(\Omega)}^{2} & =\int_{\Omega} w_{t}^{k+1} w^{k+1} d x \\
& +\int_{\Omega} \mathcal{H}\left(u^{k}\right) \nabla w^{k+1} \nabla w^{k+1} d x,
\end{aligned}
$$

de (28) y (29), resulta en particular que

$$
\begin{aligned}
& D_{0}\left\|\nabla w^{k+1}\right\|_{L^{2}(\Omega)}^{2} \\
& \quad \leq\left\|w^{k}\right\|_{L^{2}(\Omega)}\left\|w^{k+1}\right\|_{L^{2}(\Omega)}+M\left\|w^{k} \nabla u^{k}\right\|_{L^{2}(\Omega)}\left\|\nabla w^{k+1}\right\|_{L^{2}(\Omega)} .
\end{aligned}
$$


Al aplicar en (30), las desigualdades de Poincaré y Hölder respectivamente, se tiene

$$
\begin{aligned}
& D_{0}\left\|\nabla w^{k+1}\right\|_{L^{2}(\Omega)}^{2} \\
& \leq\left\|w^{k}\right\|_{L^{2}(\Omega)}\left\|w^{k+1}\right\|_{L^{2}(\Omega)}+M\left\|w^{k}\right\|_{L^{4}(\Omega)}\left\|\nabla u^{k}\right\|_{L^{4}(\Omega)}\left\|\nabla w^{k+1}\right\|_{L^{2}(\Omega)} \\
& \leq\left\|w^{k}\right\|_{L^{2}(\Omega)} C_{p}\left\|\nabla w^{k+1}\right\|_{L^{2}(\Omega)}+M\left\|w^{k}\right\|_{L^{4}(\Omega)}\left\|\nabla u^{k}\right\|_{L^{4}(\Omega)}\left\|\nabla w^{k+1}\right\|_{L^{2}(\Omega)}
\end{aligned}
$$

y puesto que $H^{1}(\Omega) \hookrightarrow L^{4}(\Omega)$ entonces

$$
D_{0}\left\|\nabla w^{k+1}\right\|_{L^{2}(\Omega)} \leq C_{p}\left\|w^{k}\right\|_{L^{2}(\Omega)}+M\left\|w^{k}\right\|_{H_{0}^{1}(\Omega)}\left\|\nabla u^{k}\right\|_{H^{1}(\Omega)}
$$

después de usar dos veces la desigualldad de Poincaré en (31), se sigue

$$
\begin{aligned}
D_{0}\left\|\nabla w^{k+1}\right\|_{L^{2}(\Omega)} & \leq C_{p}^{2}\left\|\nabla w^{k}\right\|_{L^{2}(\Omega)}+M \sqrt{C_{p}^{2}+1}\left\|\nabla w^{k}\right\|_{L^{2}(\Omega)}\left\|u^{k}\right\|_{H^{2}(\Omega)} \\
& =\left(C_{p}^{2}+M \sqrt{C_{p}^{2}+1}\left\|u^{k}\right\|_{H^{2}(\Omega)}\right)\left\|\nabla w^{k}\right\|_{L^{2}(\Omega)}
\end{aligned}
$$

Además, del Teorema 5, §7.1.3, en [5], se tiene

$$
\underset{0 \leq t \leq T}{\operatorname{ess} \sup }\left\|u^{k}\right\|_{H^{2}(\Omega)} \leq C\left(\frac{1}{4}+\left\|u_{0}\right\|_{H^{2}(\Omega)}\right)
$$

Por consiguiente

$$
\left\|\nabla w^{k+1}\right\|_{L^{2}(\Omega)}^{2} \leq C_{2}\left\|\nabla w^{k}\right\|_{L^{2}(\Omega)}^{2}
$$

donde $C_{2}:=\left(\frac{C_{p}^{2}+M \sqrt{C_{p}^{2}+1}\left\|u_{0}\right\|_{H^{2}(\Omega)}}{D_{0}}\right)^{2}$.

Por otra parte, de (28) y (29), se tiene también que

$$
\begin{aligned}
& \frac{1}{2} \frac{d}{d t}\left\|w^{k+1}\right\|_{L^{2}(\Omega)}^{2} \\
& \quad \leq\left\|w^{k}\right\|_{L^{2}(\Omega)}\left\|w^{k+1}\right\|_{L^{2}(\Omega)}+M\left\|w^{k} \nabla u^{k}\right\|_{L^{2}(\Omega)}\left\|\nabla w^{k+1}\right\|_{L^{2}(\Omega)} .
\end{aligned}
$$


Al aplicar a (34), las desigualdades de Young, Hölder, el encaje de Sobolev $H^{1}(\Omega) \hookrightarrow L^{p}(\Omega)$ para $2 \leq p<\infty$ y (33) respectivamente, se tiene

$$
\begin{aligned}
& \frac{1}{2} \frac{d}{d t}\left\|w^{k+1}\right\|_{L^{2}(\Omega)}^{2} \\
\leq & \frac{1}{2 \lambda_{0}}\left\|w^{k}\right\|_{L^{2}(\Omega)}^{2}+\frac{\lambda_{0}}{2}\left\|w^{k+1}\right\|_{L^{2}(\Omega)}^{2}+\frac{M^{2}}{2 \lambda_{1}}\left\|w^{k} \nabla u^{k}\right\|_{L^{2}(\Omega)}^{2}+\frac{\lambda_{1}}{2}\left\|\nabla w^{k+1}\right\|_{L^{2}(\Omega)}^{2} \\
\leq & \frac{1}{2 \lambda_{0}}\left\|w^{k}\right\|_{L^{2}(\Omega)}^{2}+\frac{\lambda_{0}}{2}\left\|w^{k+1}\right\|_{L^{2}(\Omega)}^{2}+\frac{M^{2}}{2 \lambda_{1}}\left\|w^{k}\right\|_{L^{4}(\Omega)}^{2}\left\|\nabla u^{k}\right\|_{L^{4}(\Omega)}^{2}+ \\
& +\frac{\lambda_{1}}{2}\left\|\nabla w^{k+1}\right\|_{L^{2}(\Omega)}^{2} \\
\leq & \frac{1}{2 \lambda_{0}}\left\|w^{k}\right\|_{L^{2}(\Omega)}^{2}+\frac{\lambda_{0}}{2}\left\|w^{k+1}\right\|_{L^{2}(\Omega)}^{2}+\frac{M^{2}}{2 \lambda_{1}} C_{3}\left\|w^{k}\right\|_{H_{0}^{1}(\Omega)}^{2}\left\|u^{k}\right\|_{H^{2}(\Omega)}^{2}+ \\
& +\frac{\lambda_{1}}{2}\left\|\nabla w^{k+1}\right\|_{L^{2}(\Omega)}^{2} \\
\leq & \left(\frac{1}{2 \lambda_{0}}+\frac{M^{2} C_{3}}{2 \lambda_{1}}\left\|u^{k}\right\|_{H^{2}(\Omega)}^{2}\right)\left\|w^{k}\right\|_{H_{0}^{1}(\Omega)}^{2}+\frac{\lambda_{0}}{2}\left\|w^{k+1}\right\|_{L^{2}(\Omega)}^{2}+ \\
& +\frac{\lambda_{1}}{2}\left\|\nabla w^{k+1}\right\|_{L^{2}(\Omega)}^{2} \\
\leq & \left(\frac{1}{2 \lambda_{0}}+\frac{M^{2} C_{4}}{2 \lambda_{1}}+\frac{\lambda_{1} C_{2}}{2}\right)\left\|w^{k}\right\|_{H_{0}^{1}(\Omega)}^{2}+\frac{\lambda_{0}}{2}\left\|w^{k+1}\right\|_{L^{2}(\Omega)}^{2},
\end{aligned}
$$

donde $C_{4}:=C_{3}\left(\frac{1}{4}+\left\|u_{0}\right\|_{H^{2}(\Omega)}^{2}\right)^{2}$ por (32).

De la desigualdad de Gronwall generalizada y (35), se obtiene

$$
\left\|w^{k+1}\right\|_{L^{2}(\Omega)}^{2} \leq\left(\left\|w^{k+1}(0)\right\|_{L^{2}(\Omega)}^{2}+C_{5}\left\|w^{k}\right\|_{H_{0}^{1}(\Omega)}^{2}\right) e^{\int \frac{\lambda_{0}}{2} d t},
$$

donde $C_{5}:=\left(\frac{1}{\lambda_{0}}+\frac{M^{2} C_{4}}{\lambda_{1}}+\lambda_{1} C_{2}\right)$.

De (33) y (36), resulta

$$
\left\|w^{k+1}\right\|_{L^{2}(\Omega)}^{2}+\left\|\nabla w^{k+1}\right\|_{L^{2}(\Omega)}^{2} \leq C_{2}\left\|\nabla w^{k}\right\|_{L^{2}(\Omega)}^{2}+C_{5}\left\|w^{k}\right\|_{H_{0}^{1}(\Omega)}^{2} e^{\int \frac{\lambda_{0}}{2} d t},
$$

es decir,

$$
\left\|w^{k+1}\right\|_{H_{0}^{1}(\Omega)}^{2} \leq\left(C_{2}+C_{5} e^{\frac{\lambda_{0}}{2} T}\right)\left\|w^{k}\right\|_{H_{0}^{1}(\Omega)}^{2} .
$$

Sea $T_{1}:=\frac{2}{\lambda_{0}} \ln \left(\frac{\frac{1}{2}-C_{2}}{C_{5}}\right)$, con $\lambda_{0}=4\left(1+M C_{4}\right)$ y $\lambda_{1}=\frac{1}{4 C_{2}}-1$. Entonces, si $0<T \leq T_{1}$,

$$
\left\|u^{k+1}-u^{k}\right\|_{L^{\infty}\left(0, T ; H_{0}^{1}(\Omega)\right)} \leq \frac{1}{2}\left\|u^{k}-u^{k-1}\right\|_{L^{\infty}\left(0, T ; H_{0}^{1}(\Omega)\right)} .
$$


Se concluye así que la sucesión $\left\{u^{k}\right\}_{k \in \mathbb{N}_{0}}$ es de Cauchy en $L^{\infty}\left(0, T ; H_{0}^{1}(\Omega)\right)$ y por consiguiente, existe una función $u \in L^{\infty}\left(0, T ; H_{0}^{1}(\Omega)\right)$ tal que

$$
u^{k} \rightarrow u \text { en } L^{\infty}\left(0, T ; H_{0}^{1}(\Omega)\right) \text { fuertemente. }
$$

Como $L^{2}\left(0, T ; H^{2}(\Omega)\right)$ y $L^{2}\left(0, T ; H_{0}^{1}(\Omega)\right)$ son espacios de Hilbert, la cota uniforme (12), implica que existe una subsucesión $\left\{u^{k_{l}}\right\}_{k_{l} \in \mathbb{N}_{0}}$ tal que

$$
\begin{aligned}
& u^{k_{l}} \rightarrow u \text { en } L^{2}\left(0, T ; H^{2}(\Omega)\right) \text { débilmente, } \\
& u_{t}^{k_{l}} \rightarrow u_{t} \text { en } L^{2}\left(0, T ; H_{0}^{1}(\Omega)\right) \text { débilmente. }
\end{aligned}
$$

Usando estas convergencias en la formulación débil de (3) y haciendo que $l \rightarrow$ $\infty$, se tiene que $u$ es solución débil del problema (1).

Ahora, supongamos que existen $u_{1}$ y $u_{2}$ soluciones débiles del problema (1), se sigue que

$$
\left\|u_{1}-u_{2}\right\|_{L^{\infty}\left(0, T ; H_{0}^{1}(\Omega)\right)} \leq \frac{1}{2}\left\|u_{1}-u_{2}\right\|_{L^{\infty}\left(0, T ; H_{0}^{1}(\Omega)\right)}
$$

para $T=T_{1}$. Por consiguiente, $u_{1}=u_{2}$ y la solución débil del problema (1), es única.

Esto completa la demostración del Teorema 1.

\section{Apéndice}

Desigualdad de Young. Para todo $a, b>0$ y $\epsilon>0$ se satisface la desigualdad

$$
a b \leq \epsilon a^{2}+\frac{b^{2}}{4 \epsilon} .
$$

Desigualdad de Gronwall. Sea $u:[0, T] \rightarrow \mathbb{R}$ una función absolutamente continua no negativa, que satisface

$$
\frac{d u}{d t}(t) \leq \phi(t) u(t)+\psi(t), \text { para casi toda } t
$$

donde $\phi(t)$ y $\psi(t)$ son funciones sumables no negativas en $[0, T]$. Entonces

$$
u(t) \leq e^{\int_{0}^{t} \phi(s) d s}\left[u(0)+\int_{0}^{t} \psi(s) d s\right]
$$

para todo $t \in[0, T]$. 
Desigualdad de Hölder. Sean $u \in L^{p}(\Omega)$ y $v \in L^{q}(\Omega)$ con $1 \leq p, q \leq \infty$ y $\frac{1}{p}+\frac{1}{q}=1$. Entonces $u v \in L^{1}(\Omega)$ y

$$
\int_{\Omega}|u v| \leq\|u\|_{L^{p}(\Omega)}\|v\|_{L^{q}(\Omega)}
$$

Desigualdad de Poincaré. Sea $\Omega \subset \mathbb{R}^{n}$ un dominio acotado con $\partial \Omega \in C^{1}$ y $u \in H_{0}^{1}(\Omega)$. Entonces existe una constante positiva $C_{p}$ tal que

$$
\|u\|_{L^{2}(\Omega)} \leq C_{p}\|\nabla u\|_{L^{2}(\Omega)} .
$$

Encajes de Sobolev. Sea $\Omega \subset \mathbb{R}^{n}$ un dominio acotado con $\partial \Omega \in C^{1}$ y $1 \leq p \leq$ $\infty$. Entonces

1. $W^{1, p}(\Omega) \subset L^{p^{*}}(\Omega)$ (continuamente) donde $\frac{1}{p^{*}}=\frac{1}{p}-\frac{1}{n}$, si $p<n$.

2. $W^{1, p}(\Omega) \subset L^{q}(\Omega)$ (continuamente) donde $q \in[p, \infty)$, si $p=n$.

3. $W^{1, p}(\Omega) \subset L^{\infty}(\Omega)$ (continuamente) si $p>n$.

Nótese que $H^{1}(\Omega):=W^{1,2}(\Omega)$.

Teorema 2 (Ver [5], Teoremas 1-4, \$7.1.2)

Sean $u_{0}(x) \in L^{2}(\Omega), f(x, t) \in L^{2}\left(0, T ; L^{2}(\Omega)\right)$ y $a(x, t) \in L^{\infty}\left(\Omega_{T}\right)$. Entonces existe una única solución débil $u \in L^{2}\left(0, T ; H_{0}^{1}(\Omega)\right) \cap L^{\infty}\left(0, T ; L^{2}(\Omega)\right)$ con $u_{t} \in L^{2}\left(0, T ; H^{-1}(\Omega)\right)$ para el problema

$$
\begin{cases}u_{t}-\nabla \cdot(a(x, t) \nabla u)=f(x, t) & \text { en } \Omega_{T} \\ u=0 & \text { en } \partial \Omega \\ u=u_{0} & \text { en } \Omega \times\{t=0\},\end{cases}
$$

en el sentido que $u(0)=u_{0} y$

$$
\int_{\Omega} u_{t} \phi d x+\int_{\Omega} a(x, t) \nabla u \nabla \phi d x=\int_{\Omega} f(x, t) \phi d x
$$

para todo $\phi \in H_{0}^{1}(\Omega)$ y casi todo $t \in[0, T]$. 
Teorema 3 (Ver [5], Teorema 5, \$7.1.3)

Suponga que $u_{0}(x) \in H^{2}(\Omega), f_{t}(x, t) \in L^{2}\left(0, T ; L^{2}(\Omega)\right)$ y $a(x, t) \in L^{\infty}\left(\Omega_{T}\right)$. Sea $u \in L^{2}\left(0, T ; H_{0}^{1}(\Omega)\right)$ con $u_{t} \in L^{2}\left(0, T ; H^{-1}(\Omega)\right)$ solución débil del problema (40). Entonces

$$
\begin{aligned}
u & \in L^{\infty}\left(0, T ; H^{2}(\Omega)\right) \\
u_{t} & \in L^{\infty}\left(0, T ; L^{2}(\Omega)\right) \cap L^{2}\left(0, T ; H_{0}^{1}(\Omega)\right) \\
u_{t t} & \in L^{2}\left(0, T ; H^{-1}(\Omega)\right)
\end{aligned}
$$

$y$

$$
\begin{array}{r}
\underset{0 \leq t \leq T}{\operatorname{ess} \sup }\left(\|u(t)\|_{H^{2}(\Omega)}+\left\|u_{t}\right\|_{L^{2}(\Omega)}\right)+\left\|u_{t}\right\|_{L^{2}\left(0, T ; H_{0}^{1}(\Omega)\right)}+\left\|u_{t t}\right\|_{L^{2}\left(0, T ; H^{-1}(\Omega)\right)} \\
\leq C\left\|u_{0}\right\|_{H^{2}(\Omega)}+\|f\|_{H^{1}\left(0, T ; L^{2}(\Omega)\right)}
\end{array}
$$

$\operatorname{con} C=C\left(\Omega, T,\|a\|_{L^{\infty}\left(\Omega_{T}\right)}\right)$.

\section{Agradecimientos}

Los autores agradecen a los árbitros anónimos por sus valiosos comentarios y sugerencias que contribuyeron para mejorar el manuscrito.

\section{References}

[1] Bertsch, M.; Gurtin, M. E.; Hilhorst, D.; Peletier, L. (1984) "On interacting populations that disperse to avoid crowding: The effect of a sedentary colony", Journal Mathematical Biology 19: 1-12.

[2] Ciarlet, P.G. (2013) Linear and Nonlinear Functional Analysis with Applications Society for Industrial \& Applied Mathematics, New York.

[3] Deutray, R.; Lions. J-L. (2000) Mathematical Analysis and Numerical Methods for Science and Tecnology, Vol. 4-6. Springer-Verlag, Heidelberg.

[4] DiBenedetto, E. (1993) Degenerate Parabolic Equations. Springer-Verlag, New York.

[5] Evans, L.C. (2010) Partial Differential Equations. American Mathematical Society, Providence, Rhode Island.

[6] Fisher, R.A. (1937) "The wave of advance of advantageous genes", Annals of Eugenics 7: 353-369. 
[7] Gurtin, M.; MacCamy, M.(1977) "On the diffusion of biological populations", Mathematical Biosciences 33: 35-49.

[8] Murray, J.D. (2003) Mathematical Biology II. Spatial Models and Biomedical Applications. Springer-Verlag, New York.

[9] Petrovskii, S.V.; Bai-Lian, L. (2006) Exactly Solvable Models of Biological Invasion. Chapman \& Hall/CRC, Boca Raton FL.

[10] Shigesada, N.; Kawasaki, K.; Takeda, Y. (1995) "Modeling stratified diffusion in biological invasions", The American Naturalist 146(2): 229-251.

[11] Shigesada, N.; Kawasaki, K. (1997) Biological Invasions: Theory and Practice. Oxford University Press, New York.

[12] Showalter, R.E. (1997) Monotone operators in Banach space and nonlinear partial differential equations. Mathematical Surveys and Monographs 49, American Mathematical Society, Providence RI.

[13] Taira, K. (2016) Analytic Semigroups and Semilinear Initial Boundary Value Problems. Part of London Mathematical Society Lecture Note Series, 2nd Edition.

[14] Tröltzsch, F. (2010) Optimal Control of Partial Differential Equations Theory, Methods and Applications. Graduate Studies in Mathematics, Vol. 112. American Mathematical Society, Providence RI.

[15] Vázquez, J.L. (2007) The Porous Medium Equation. Oxford University Press, Oxford.

[16] Witelsky, T.P. (1995) "Merging traveling waves for the porous-Fisher's equation", Applied Mathematics Letters 8(4): 57-62

[17] Zeidler, E. (1990) Nonlinear Functional Analysis and its Applications II/B: Nonlinear Monotone Operators. Springer-Verlag, New York. 